\title{
NCL: Storing ambient settings in media node
}

\author{
Fábio Barreto \\ MídiaCom Lab - UFF \\ UNILASALLE-RJ \\ fbarreto@midiacom.uff.br
}

\author{
Eyre Brasil B. Montevecchi \\ MídiaCom Lab - UFF \\ eyrebrasil@midiacom.uff.br
}

\author{
Raphael Abreu \\ MídiaCom Lab - UFF \\ raphael.abreu@midiacom.uff.br
}

\author{
Joel A. F. dos Santos \\ CEFET/RJ \\ jsantos@eic.cefet-rj.br
}

\author{
Débora C. Muchaluat-Saade \\ MídiaCom Lab - UFF \\ debora@midiacom.uff.br
}

\begin{abstract}
This proposal consists of adding one more type of variable storage setting node, called "x-ncl-ambient-settings," which stores environment information. This information may come from sensors in the physical ambient that must update this data periodically or as defined by the author of the NCL document. These variables can be used by NCL links or procedural code to drive application behavior based on the perceived environment information.
\end{abstract}

\section{KEYWORDS}

NCL, ambient settings, IoT, authoring

\section{BACKGROUND}

Desenvolvimentos em computação ubíqua, sensores, atuadores e o advento da IoT (Internet Of Things) têm o potencial de mudar a forma como os consumidores interagem com a tecnologia. Aprimoramentos em dispositivos sensores e atuadores permitem um maior nível de interação entre os ambientes das aplicações e seus usuários. Isso representa uma oportunidade interessante para sistemas multimídia a fim de expandir possibilidades de interação com o usuário, possibilitando aplicações interativas mais imersivas. Assim, se torna interessante prover para o autor de conteúdo novas construções em linguagens de autoria declarativas para que ele possa desenvolver aplicações contemplando conteúdos lidos de dispositivos sensores do ambiente físico. Essa é uma abordagem que permite ao autor da aplicação considerar o estado do ambiente para a definição do comportamento da aplicação ou sua adaptação. Para viabilizar esta funcionalidade é necessária a existência de um nó em NCL que armazene informações lidas do ambiente e associe-as a eventos para que a mudança de comportamento da aplicação seja associada a atributos variáveis deste ambiente.

\section{PROPOSAL}

Conforme apresentado na norma ABNT NBR15606-2, apenas é permitido o armazenamento de variáveis em NCL por meio do nó $<$ media > do tipo application/x-ncl-settings. Estas podem ser globais definidas pelo autor ou variáveis de ambiente reservadas para o sistema GINGA. Porém os valores de propriedades do nó do tipo application/X-ncl-settings podem apenas ser modificados através do uso de elos NCL. Isto inviabiliza que um código procedural

In: Future of Interactive Television Workshop (V WTVDI), Rio de Janeiro, Brasil. Anais Estendidos do Simpósio Brasileiro de Sistemas Multimídia e Web (WebMedia). Porto Alegre: Sociedade Brasileira de Computação, 2019.

ISSN 2596-1683 (tal como a leitura de um sensor) atualize os valores destes dados. Além disso, só é permitido um nó do tipo ncl-settings em cada documento NCL. Neste contexto, este documento propõe adicionar um tipo de mídia denominado application/x-ncl-ambient-settings, que armazena informações do ambiente físico em que a aplicação está executando. O novo tipo tem cardinalidade maior que 1 , assim como os demais tipos de nó <media $>$. As informações armazenadas podem ser advindas de sensores que deverão atualizar esses dados periodicamente ou de forma definida pelo autor do documento NCL.

Todos os sensores que provêem dados para um nó de mídia do tipo x-ncl-ambient-settings podem estar associados a uma posição no espaço. $\mathrm{O}$ atributo descriptor de um nó $\mathrm{x}$-ncl-ambient-settings pode referenciar o elemento region no qual delimita a região do ambiente em que se deseja ler informações. Esta região pode ser definida por eixos x, y e z, definidos no padrão MPEG-V [1], ou por coordenadas esféricas, assim como definido em Josué et al. [2]. As coordenadas esféricas também devem conter os parâmetros de largura e altura para indicar o tamanho da região da qual deseja-se obter dados de sensores. A partir do momento em que o sensor é encontrado na região definida, seus dados podem ser armazenados. Quando houver mais de um sensor do tipo desejado na região, o critério de escolha pode ser uma média aritmética, menor valor ou maior valor de leitura, a critério do formatador. Os tipos de informações coletadas serão importados do padrão MPEG-V. Se nenhuma região for definida, um sensor em qualquer posição no ambiente físico pode ser utilizado.

A Listagem 1 apresenta um exemplo da utilização de ambientsettings. Na listagem, nenhuma região é definida, portanto, sensores em qualquer posição do ambiente podem ser utilizados. É definido um nó x-ncl-ambient-settings contendo propriedade que indica os tipos de sensores a serem utilizados, luminosidade e temperatura.

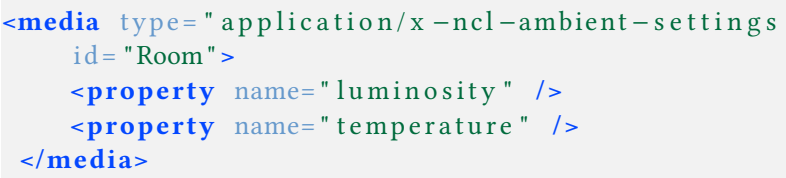

Listagem 1: Especificação de nós do tipo ambient-settings

\section{USE CASE(S)}

Nesta seção será apresentado um caso de uso para ilustrar a utilização de ambient-settings para armazenar dados de sensores e direcionar o comportamento de uma aplicação. O caso de uso em questão é de uma aplicação de uma exposição de arte interativa. 
A aplicação apresenta uma mídia na TV e, em um determinado momento, exibe um vídeo dependendo da proximidade do usuário. Para isto, a aplicação conta com um sensor de proximidade em frente à TV. Caso o sensor de proximidade alcance um certo limiar de $50 \mathrm{~cm}$ (i.e., alguém se aproximou), a mídia proximo.mp4 será executada. A Listagem 2 apresenta um trecho da especificação em NCL para realizar tal aplicação.

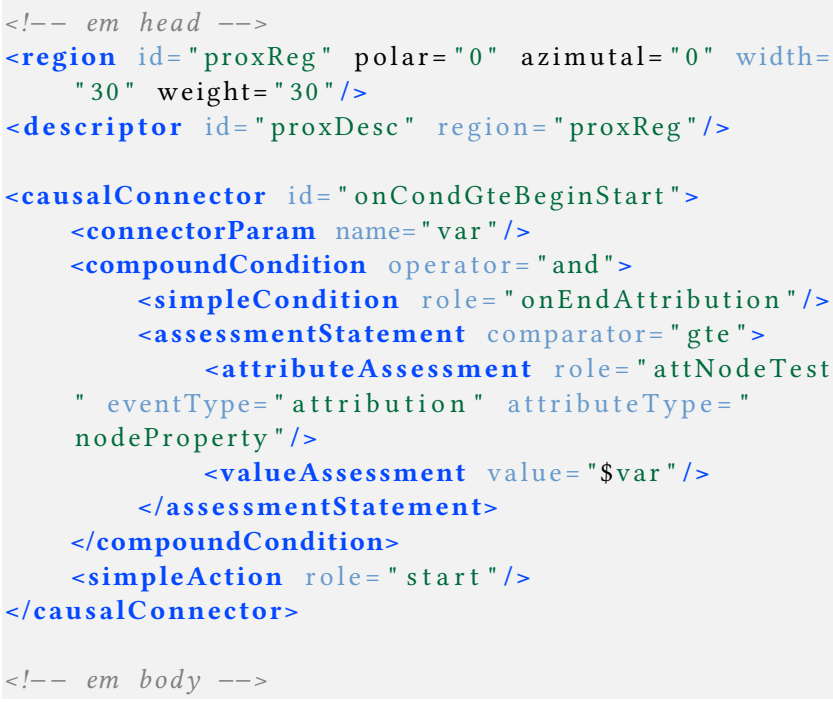

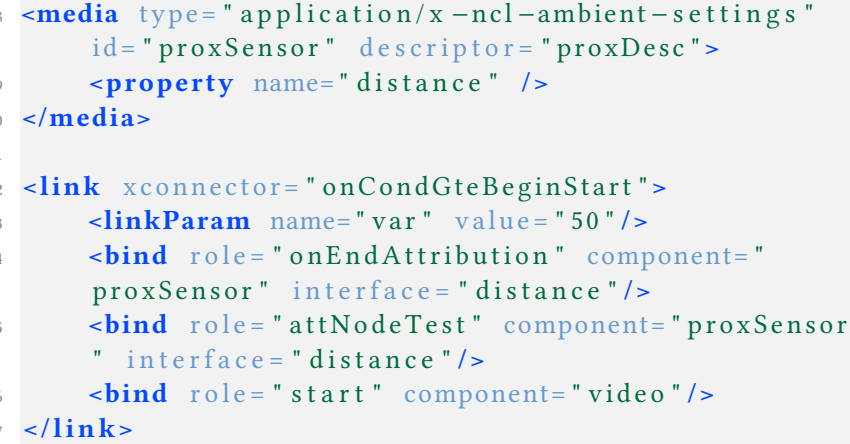

Listagem 2: Trecho de aplicação em NCL utilizando nó do tipo ambient-settings

\section{ACKNOWLEDGMENTS}

Os autores gostariam de agradecer a CAPES, CNPq e FAPERJ pelo apoio financeiro parcial deste trabalho.

\section{REFERENCES}

[1] ISO/IEC 23005-1 2016. Information technology - Media context and control - Part 1: Architecture. Standard. International Organization for Standardization, Geneva, $\mathrm{CH}$.

[2] Marina Josué, Raphael Abreu, Fábio Barreto, Douglas Mattos, Glauco Amorim, Joel dos Santos, and Débora Muchaluat-Saade. 2018. Modeling Sensory Effects As First-class Entities in Multimedia Applications. In Proceedings of the 9th ACM Multimedia Systems Conference (MMSys '18). ACM, New York, NY, USA, 225-236. https://doi.org/10.1145/3204949.3204967 